\title{
Cortisol response to psychological stress in patients with schizophrenia in the Chinese population
}

\section{Xiaoyu Zhu}

Beijing HuiLongGuan Hospital

\section{Yu Zhu}

Beijing HuiLongGuan Hospital

Junchao Huang

Beijing HuiLongGuan Hospital

Yanfang Zhou

Beijing HuiLongGuan Hospital

Jinghui Tong

Beijing HuiLongGuan Hospital

Ping Zhang

Beijing HuiLongGuan Hospital

Xingguang Luo

Yale University

\section{Song Chen}

Beijing HuiLongGuan Hospital

\section{Baopeng Tian}

Beijing HuiLongGuan Hospital

\section{Shuping Tan}

Beijing HuiLongGuan Hospital

\section{Zhiren Wang}

Beijing HuiLongGuan Hospital

\section{Xiaole Han}

Beijing HuiLongGuan Hospital

\section{Li Tian}

University of Tartu

Chiang-Shan Li

Yale University

\section{Elliot Hong}

University of Maryland, Baltimore

Yunlong Tan ( $\nabla$ yltan21@126.com ) 
Beijing HuiLongGuan Hospital

\section{Research Article}

Keywords: Cortisol stress response, Distress intolerance, Hypothalamic-pituitary-adrenal (HPA) axis, Schizophrenia

Posted Date: February 16th, 2022

DOl: https://doi.org/10.21203/rs.3.rs-702720/v2

License: (a) (i) This work is licensed under a Creative Commons Attribution 4.0 International License. Read Full License 


\section{Abstract}

Accumulating evidence suggests that hypothalamic-pituitary-adrenal (HPA) axis dysfunction might play an important role in the pathophysiology of schizophrenia. This study aimed to explore the cortisol response to psychological stress in patients with schizophrenia. In this study, patients with schizophrenia $(n=104)$ and healthy volunteers $(n=59)$ were asked to complete psychological stress challenge tasks, which included the Paced Auditory Serial Addition Task and Mirror-Tracing Persistence Task, and then, saliva was collected to measure cortisol levels. Emotions and psychopathology were assessed by the Positive and Negative Affect Schedule (PANAS) and Positive and Negative Syndrome Scale (PANSS). The results showed (1) that the cortisol stress response and negative emotions in patients with schizophrenia differed significantly from those in healthy controls; (2) there were significant interactions between sampling time and diagnosis for saliva cortisol levels; (3) there were significant interactions between scoring time and diagnosis for the negative affect score of the PANAS; and (4) the changes in salivary cortisol levels and negative affect scores before and after the psychological stress challenge tasks were not correlated with clinical symptoms in patients with schizophrenia. These findings indicated that an abnormal cortisol stress response might be a stable biological characteristic of schizophrenia.

\section{Introduction}

Schizophrenia is a severe neuropsychiatric disorder for which the etiology remains unclear. However, the interaction of multiple biological and environmental factors plays an important role in the pathophysiology of this illness. Several models of stress-vulnerability have been proposed for schizophrenia ${ }^{[1-4]}$ to better understand the relationships between vulnerability, stress, and disease. The hypothalamic-pituitary-adrenal (HPA) axis is one of the primary systems that moderate the physiological response to psychological and physiological stressors ${ }^{[5]}$, and cortisol secretion levels can reflect the function of the HPA axis.

Cortisol secretion is indexed using morning cortisol levels, diurnal cortisol levels, cortisol awakening response (CAR), and cortisol stress response. Morning cortisol levels reflect basal HPA axis function, diurnal cortisol levels reflect the fluctuation of the HPA axis throughout the day ${ }^{[6]}$, and CAR and cortisol stress response reflect the mobilization capability and response to stress of the HPA axis, as awakening can be considered a stressor ${ }^{[7,8]}$. The effective cortisol response to stress is marked by a rapid rise in cortisol levels right after the introduction of the stressor, followed by a rapid decline. This physiological process plays a key role in the body's ability to adapt to the environment and deal with threats ${ }^{[9]}$. Cortisol stress response is an important indicator used to evaluate HPA axis function.

The association between cortisol and schizophrenia has been widely recognized ${ }^{[9]}$. In general, patients with schizophrenia have abnormally elevated cortisol levels, but the results of current studies are

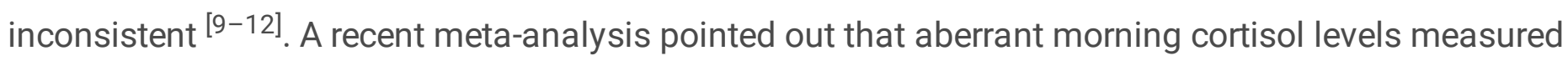
peripherally (blood or saliva) were observed more frequently in patients with schizophrenia than in controls; however, they might have been influenced by factors such as illness stage, mood-state, and 
psychotropic medication ${ }^{[10]}$. Studies have also noted a blunted CAR in patients with schizophrenia ${ }^{[13-}$

15]. Although antipsychotic treatment can alleviate the effects of abnormal diurnal cortisol levels ${ }^{[4,16]}$, the blunted CAR persists ${ }^{[17]}$. These results suggest that changes in cortisol levels might be a more stable indicator.

In addition to CAR, another indicator of changes in cortisol levels is cortisol stress response. The cortisol response to psychosocial stress is reportedly observed but blunted in patients with schizophrenia when compared to that in controls ${ }^{[9]}$. Girshkin et al. reported patients with schizophrenia did not show any cortisol increase in response to magnetic resonance imaging (MRI) as a stressor ${ }^{[12]}$, unlike healthy individuals ${ }^{[18]}$. This differed from the cortisol stress response shown among patients with schizophrenia in most studies using laboratory-based psychological stress challenge tasks ${ }^{[9,11]}$. One possible explanation for this was that the stress during an MRI mainly involved passive perception of noise and did not require the patient to participate in the execution; it was merely an uncomfortable environment, and a lack of concern about the environment is a manifestation of apathy in patients with schizophrenia.

Other studies also found heightened cortisol secretion or flattened CAR among individuals who could not be diagnosed with schizophrenia but who met the clinical high-risk (CHR) criteria for psychosis when compared to that in healthy controls ${ }^{[7,19-22]}$. Similar results have been reported among relatives of patients with schizophrenia ${ }^{[21-23]}$. These findings suggest abnormal cortisol secretion predates the onset of psychiatric symptoms and may be genetic. Cortisol-related abnormalities and the HPA axis dysfunction they represent may serve as biomarkers for predicting the occurrence and development of schizophrenia.

In this study, we aimed to investigate the difference in cortisol response to psychological stress between individuals with schizophrenia and healthy controls in the Chinese population and to test the hypothesis that patients with schizophrenia had an abnormal cortisol response to stress, which represents dysregulated HPA function, and the HPA axis dysfunction has the potential to serve as a biomarker for schizophrenia. Salivary cortisol secretion is a reliable indicator of free cortisol in plasma and has been used as an index for HPA axis activity in many studies ${ }^{[24]}$, thus we noninvasively examined cortisol levels by testing saliva collected before and after a laboratory-based psychological stress challenge task.

\section{Material And Methods 2.1 Participants}

Patients were recruited from inpatients or outpatients at the Beijing HuiLongGuan Hospital from 2017 to 2019. The inclusion criteria for patients were as follows: (1) diagnoses confirmed according to the Structured Clinical Interview in the Diagnostic and Statistical Manual of Mental Disorders (DSM)-IV diagnostic criteria for schizophrenia; (2) age between 18 and 60 years; and (3) Han Chinese ancestry. 
Patients were excluded if they had: (1) evidence of a significant physical illness or neurological condition; (2) substance dependence or current substance abuse (except nicotine) at the time of screening; (3) a psychiatric disorder other than schizophrenia according to DSM-IV criteria; (4) any endocrine disorder; or (5) received any immunotherapies.

Age- and sex-matched healthy volunteers were recruited from the local community. Healthy volunteers were excluded if they had (1) evidence of a significant physical illness or neurological condition; (2) current and ongoing substance abuse (except nicotine) at the time of screening; (3) any psychiatric disorder according to the DSM-IV criteria; (4) any endocrine disorder; or (5) received any immunotherapies.

\subsection{Psychological stress challenge task}

We used a psychological stress challenge task to assess the capability of participants to persist in goaldirected behavior while under stress. The task consisted of two tests each lasts for 7 minutes: the Paced Auditory Serial Addition Task (PASAT) and the Mirror-Tracing Persistence Task (MTPT), both of which have been applied in psychiatric research ${ }^{[25]}$. The task is monotonous, and there is a danger of being punished. This makes the task somewhat difficult, so some participants may choose to drop out. During the PASAT, multiple numbers are displayed in sequence on a computer screen; the participant is asked to sum the numbers that appear and then use the mouse to select the correct answer. When the participant answers incorrectly, the computer plays annoying sounds. The computer program adjusts the number display speed based on the participant's performance to ensure that the test is challenging. During the MTPT, participants are asked to use the mouse to trace the outline of a star on the computer screen. However, the moving direction of the cursor on the screen is opposite to that of the mouse in the participant's hand, creating a challenge. If the cursor leaves the track or stands still, it is judged as an error, causing the computer to play annoying noises and reset the drawing process. The computer adjusts the width of the star according to the participant's performance to ensure the task is challenging for everyone.

Since these two tasks involve two different skills (computing and hand-eye coordination), their combination helps avoid biases that may be caused by individual expertise. The two tasks were performed sequentially and in random order. The whole process was completed in the form of humancomputer dialogue, thus avoiding the subjective influence of researchers.

Participants could opt-out at any time during the task. They were told in advance that they would be compensated for participating. Participants received 40 Yuan for completing both tasks. If they did not complete the tasks, the compensation was halved.

We determined distress tolerance when a participant persisted in completing either of the tasks; we determined distress intolerance when a participant failed to complete both tasks.

\subsection{Saliva collection and processing}


To reduce the effects of changes in cortisol over time, all tasks and saliva sample collection were held between 12:00 and 4:00pm. We collected three saliva samples from each participant: before starting the tasks and after resting for 20 and 40 minutes following the tasks. To reduce interference factors, all participants were asked not to do strenuous exercise, eat, drink, smoke, or brush their teeth for 1 hour before the test, and then to gargle 10 minutes before the test.

Saliva samples were collected using a Salivette device (Sarstedt AG \& Co Kommanditgesellschaft SarstedtstraBe 151588 Nümbrecht Germany). Participants were asked to chew the swabs thoroughly for 2 minutes at each sample collection time to make sure more than $1 \mathrm{~mL}$ of saliva was collected. The saliva sample was then frozen at $-20^{\circ} \mathrm{C}$ for 24 hours. After thawing the frozen saliva, it was centrifuged at $4^{\circ} \mathrm{C}$ for 10 minutes $(4,000 \mathrm{rpm} / \mathrm{min})$ to obtain more than $0.5 \mathrm{~mL}$ saliva from each sample. Then, the processed saliva sample was placed into a centrifuge tube; the internal standard solution was added, followed by the precipitant; and the tube was vortexed and centrifuged for 10 minutes. The supernatant was then taken out and blown dry with nitrogen. Derivatization reagent was added to the dried sample, and this was placed in the oven for derivatization. After derivatization, the supernatant was used for liquid chromatography and tandem mass spectrometry using standard protocols. The intra-trial coefficient of variations for high-, medium-, and low- quality of salivary cortisol were $8.8 \%, 5.6 \%$, and $10.1 \%$, respectively.

\subsection{Clinical assessment}

All participants completed the Positive and Negative Affect Schedule (PANAS) ${ }^{[26]}$ before the start of the first task, after completing (or aborting) the first task, and after completing (or aborting) the second task. This scale contains ten positive affect and ten negative affect (NA) items. Considering the stress response mainly involves negative emotions, we only used the NA scores.

A total of 176 participants, including 112 patients with schizophrenia and 64 healthy volunteers, completed the psychological stress challenge task, PANAS, and saliva collection. Psychopathology was assessed by an attending psychiatrist using the Positive and Negative Syndrome Scale (PANSS) ${ }^{[27]}$. The process is shown in Figure 1.

\subsection{Statistical Analyses}

The saliva cortisol data collected in the patient and control groups were analyzed. Data that deviated from the $25 \%-75 \%$ range for each set in each group of data by more than three times the interquartile range (IQR) of the set were defined as extreme values and excluded from the final analysis. Finally, the data of 104 patients and 59 controls were included.

Salivary cortisol data and PANAS score data are presented as the mean and standard deviation. A oneway analysis of variance (ANOVA) was used to examine the impacts of each factor on variables, and a repeated-measures ANOVA was used to examine the interaction between multiple factors, such as diagnosis (patients or controls), sampling time (before, and 20 minutes and 40 minutes after testing), and distress intolerance (yes or no) on variables. The chi-squared test was used to assess differences in 
categorical variables between groups. Spearman's rank correlation coefficient was also used to examine the correlation between variables. Nonparametric tests were used to examine group differences for dependent variables that did not exhibit normal distributions. The significance level was set at $5 \%(p<$ 0.05).

\subsection{Ethical statement}

This research follows the 1964 Declaration of Helsinki and subsequent amendments, or similar ethical standards. This research was approved by the Human Research Ethics Committee of Beijing HuiLongGuan Hospital [Protocol No. 2017-49]. Written informed consent was obtained from all subjects.

\section{Results}

\subsection{Participant characteristics}

In terms of age, sex, smoking status, education level, and PASAT error rate, there were no significant differences between the patients with schizophrenia and the healthy controls. The error rate of MTPT in the patients with schizophrenia was significantly higher than that of the controls. In terms of stress tolerance, there was a significant difference between the patients with schizophrenia and the controls (Table 1).

Table 1. Participant demographics and clinical characteristics

\begin{tabular}{lllll} 
& $\begin{array}{l}\text { Patients } \\
(n=104)\end{array}$ & $\begin{array}{l}\text { Healthy controls } \\
(n=59)\end{array}$ & $\begin{array}{l}\text { Statistics }(\mathrm{z} / \\
\left.\chi^{2}\right)\end{array}$ & $\begin{array}{l}P \\
\text { value }\end{array}$ \\
\hline Age, mean (SD) & $40.43 \pm 12.67$ & $37.42 \pm 12.72$ & -1.444 & 0.149 \\
\hline Sex (Male \%) & $59(56.7 \%)$ & $34(57.6 \%)$ & 0.012 & 0.912 \\
\hline Distress intolerance (DI \%) & $18(17.3 \%)$ & $1(1.7 \%)$ & 8.911 & 0.003 \\
\hline $\begin{array}{l}\text { PASAT error rate, mean } \\
\text { (SD) }\end{array}$ & $0.161 \pm 0.132$ & $0.149 \pm 0.109$ & -1.720 & 0.085 \\
\hline MTPT error rate, mean (SD) & $0.712 \pm 0.288$ & $0.309 \pm 0.199$ & -8.148 & $<0.001$ \\
\hline $\begin{array}{l}\text { Smoking status (smokers } \\
\%)\end{array}$ & $32(30.8 \%)$ & $13(22.0 \%)$ & 1.437 & 0.231 \\
\hline Education (years) & $12.63 \pm 3.75$ & $13.08 \pm 2.57$ & 0.492 & 0.643
\end{tabular}

PASAT, Paced Auditory Serial Addition Task; MTPT, Mirror-Tracing Persistence Task.

\subsection{Analyses of cortisol levels}

We found no significant interaction between sampling time and $\operatorname{sex}(F=0.032, p=0.951)$ or sampling time and smoking status $(F=0.350, p=0.669)$. The main effect of both $\operatorname{sex}(F=3.348, p=0.069)$ and 
smoking status $(F=0.368, p=0.545)$ were not significant. Using age as a covariate, the above results remained valid.

For ease of description, we assigned subscripts to the variables of salivary cortisol data collected at different times: 1 represented data before the task, 2 represented data 20 minutes after the task, and 3 represented data 40 minutes after the task.

In the patient group, saliva cortisol levels first increased and then decreased. In the control group, saliva cortisol levels were highest before the task began, and then gradually decreased (Figure 2A).

There was a significant interaction between sampling time and diagnosis $(F=6.961, p=0.002)$. Using the NA score before the task as covariates, the above results remained valid. To exclude the influence from the interaction, we used one-way ANOVA to examine the impacts of sampling time and diagnosis on the salivary cortisol levels of the two groups separately. Before the task, the saliva cortisol level of the control group tended to be higher than that of the patient group $\left(F_{1}=3.875, P_{1}=0.051\right)$, but there were no significant differences between the two groups 20 minutes $\left(F_{2}=0.261, p_{2}=0.610\right)$ or 40 minutes $\left(F_{3}=\right.$ $\left.1.361, p_{3}=0.245\right)$ after the task. The main effect of sampling time on the patient group was not significant $(F=1.250, p=0.287)$, while the main effect of sampling time on the control group was significant $(F=13.383, p<0.001)$. This indicated the stress response of cortisol during the psychological stress challenge task test was blunt among patients with schizophrenia and more active among the healthy controls.

\subsection{NA scores}

For ease of description, we assigned subscripts to the variables of NA scores evaluated at different times: 1 represented data before the task, 2 represented data after one task, and 3 represented data after both tasks.

There was a significant interaction between scoring time and diagnosis $(F=14.744, p<0.001)$. To exclude the influence from the interaction, we used one-way ANOVA to examine the impacts of scoring time and diagnosis on the NA scores of the two groups separately. The NA scores of the patients with schizophrenia were significantly higher before the task $\left(F_{1}=18.442, P_{1}<0.001\right)$ than those of the controls. There was no significant difference between patients and controls in the NA scores after completing one task $\left(F_{2}=0.006, p_{2}=0.937\right)$ and the NA scores after completing two tasks $\left(F_{3}=0.066, p_{3}\right.$ $=0.798)$. The main effect of scoring time in the patient group was not significant $(F=0.307, p=0.712)$, while the main effect of scoring time in the control group was significant $(F=16.168, p<0.001)$. These results indicated that patients with schizophrenia had more negative emotions before the test, while the psychological stress challenge task test had no significant effect on this experience. The controls had fewer negative emotions before the test, and their negative emotions increased during the test (Figure 2B). 
In the control group, most subjects persisted in completing the task, and only one subject chose to drop out (showed distress intolerance). The rate of distress intolerance in the schizophrenia group was significantly higher (Figure 3A).

As there was only one participant who showed distress intolerance in the control group, further grouping and comparison were not necessary. Therefore, we only divided the patients with schizophrenia into subgroups based on stress tolerance and made comparisons among the subgroups.

Although the trends of cortisol-level change differed between the subgroups, there was no significant interaction between sampling time and tolerance $(F=1.985, p=0.145)$ and the main effects of sampling time or tolerance were not significant $(F=2.991, p=0.058$ and $F=0.017, p=0.897$, respectively) (Figure 3B).

In the NA score, there was a significant interaction between scoring time and tolerance $(F=4.338, p=$ $0.019)$, but the main effects of scoring time $(F=2.604, p=0.083)$ and tolerance $(F=0.180, p=0.672)$ were not significant (Figure 3C).

\subsection{Clinical symptoms}

The PANSS positive subscale scores, general psychopathological subscale scales, and total scores differed significantly between patients who showed distress tolerance and those who did not. A higher score for the distress-intolerance group indicated that these patients' symptoms may be more severe (Figure 3D).

We performed a correlation analysis of the total PANSS scores, positive subscale scores, negative subscale scores, general psychopathological subscale scales, and salivary cortisol level of the patients with schizophrenia. Most of the data showed no significant correlations. The saliva cortisol level before the task was correlated with negative subscale scores $(r=0.233, p=0.017)$. The cortisol level 20 minutes after the task was correlated with PANSS total scores $(r=0.198, p=0.044)$ and negative subscale scores $(r=0.277, p=0.004)$. However, mathematically speaking, the correlation is generally considered meaningful only if the correlation coefficient is greater than 0.3 ; the above correlation coefficients were all too low, indicating no practical significance. There was no correlation between patients' salivary cortisol level 40 minutes after the task and the PANSS total scores, negative subscale scores, positive subscale scores, or general psychopathological subscale scales. None of the PANSS scales were correlated with the patients' NA scores.

\subsection{Rigor}

We reran the analyses including the data of the 13 participants with extreme values that were previously excluded, and this did not change the outcome.

\section{Discussion}




\subsection{Cortisol and schizophrenia}

Changes in saliva cortisol level before and after stress represent the function of the cortisol stress response and HPA axis. The previous stress-vulnerability model theoretically proposed the relationship between HPA axis function and schizophrenia ${ }^{[1-4]}$, which has been verified in several studies ${ }^{[9]}$. In this study, the existence of time and diagnosis interactions showed that the trends in the cortisol level change of the two groups differed greatly as the psychological stress challenge task progressed. The patients' cortisol stress response was not obvious: although it tended to first rise and then fall, the change was not significant $(p>0.05)$. The overall response was flattened, which was similar to the blunted CAR previously reported in patients with schizophrenia ${ }^{[13-15,17]}$. The change trend of salivary cortisol levels in the control group differed greatly from that of the patient group; the salivary cortisol level was clearly high before the task began, and then gradually decreased. The difference in cortisol stress response between patients with schizophrenia and healthy controls was obvious, confirming the hypothesis that patients with schizophrenia had HPA axis dysfunction.

\subsection{Cortisol, stress, and distress intolerance}

We chose to measure cortisol stress response, as it better reflects the pathological process than a baseline cortisol level ${ }^{[9]}$.

Prior to the start of the psychological stress challenge task, the baseline cortisol level increased due to the effects of anticipation anxiety. Although our study did not take samples at the same time another day for comparison, a previous study performed this comparison, confirming the existence of the expected anxiety ${ }^{[25]}$. We found pre-task cortisol levels were significantly higher than cortisol levels after the task in the controls, which was inconsistent with the findings of previous studies in an American population [11]. Since we controlled the same initial experimental conditions for all participant, such as experimenting at the same time in the afternoon, avoid strenuous exercise, eating, drinking, smoking, brushing teeth, and other actions that may affect cortisol test values before the experiment, we believe that cortisol levels represent stress levels, so a high level of cortisol suggests that healthy controls already had significant sensations of stress before the task began. Considering that previous studies were conducted in the Western parts of the world, some differences may have been due to our participants being from a Han Chinese population. Many studies revealed racial differences in cortisol levels ${ }^{[28-31]}$, one study also found that people of different ethnicity have different responses to psychological support under pressure ${ }^{[32]}$. All these suggest that cortisol and stress-related research may have ethnic differences. But there are no specific conclusions for racial differences in the effects of expected anxiety on cortisol levels yet. This point warrants further study.

The influence of culturally related psychological factors is another possible reason for the differences. Studies have shown that cultural differences between the East and the West affect people's attitudes toward examinations. In some cultural atmospheres that emphasize examinations, test anxiety is more obvious and common ${ }^{[33,34]}$; China has such a cultural atmosphere, and compared with participants in 
previous Western studies ${ }^{[11,25]}$, our subjects had a lower task withdrawal rate, which may have also reflected this cultural atmosphere. After learning they would take a test, the healthy controls might have had higher expectations for their performance, which could also have caused severe anticipation anxiety. However, the patients felt less stressed before the task, which is consistent with the apathy and decreased self-expectation caused by schizophrenia.

Cortisol levels in both groups showed a downward trend after the 40-minute rest following the task, which is a normal part of stress response. However, the patients' cortisol level decreased at a much slower rate. The cortisol level of the patient group before the task was significantly lower than that of the controls, while the cortisol level $\mathbf{4 0}$ minutes after the end of the task tended to be higher than that of the controls, indicating the response of the patients' HPA axis was insensitive or delayed, or that the negative feedback mechanism was damaged and unable to escape from stress. This finding mirrors earlier studies, where some patients showed a significant delay in cortisol stress response ${ }^{[11]}$.

Among the patients, the trends of changes in cortisol levels in distress tolerance and distress-intolerance patients were different. Among patients with distress intolerance, there was an upward trend in cortisol levels during the task, and then they dropped more sharply after the task. This may indicate that these patients felt that the pressure during the task greatly increased and exceeded their tolerance threshold, leading them to opt-out. When they decided to give up, most of the stress disappeared, and cortisol levels dropped rapidly. However, patients with distress tolerance may have been insensitive to the stress caused by the task, leading to the indifference of the process for completing the task and a smoother curve in the change of their cortisol levels. As this study was limited by a small sample size of patients with distress tolerance, our conclusions warrant further verification.

\subsection{Clinical symptoms and other indicators}

We found that cortisol stress response was not significantly correlated to patient symptoms, nor was it correlated with negative emotion scores before the task. Education level and smoking status had no significant effect on cortisol stress response, which is consistent with previous research ${ }^{[11,12]}$.

Although there are few studies on cortisol stress response in patients with schizophrenia, there are some studies on other cortisol indicators, and the results are inconsistent. Most of them found no significant correlation between cortisol and symptoms ${ }^{[35-37]}$, with similar findings for CHR patients ${ }^{[7,19]}$. However, a study of treatment-resistant patients with schizophrenia found that patients' serum cortisol levels were significantly reduced in the morning after treatment, and, although the correlation was weak, it was related to improvement in PANSS negative symptoms ${ }^{[38]}$. One study also found that, in chronic schizophrenia, cortisol levels were weakly correlated with the PANSS negative-symptom subscale ${ }^{\text {[39] }}$. Another study found that, after treatment, the increase in cortisol during the day in patients with schizophrenia was relieved, but the blunted CAR did not change ${ }^{[17]}$. These differences may have been due to varying research methods. Previous studies showed that symptoms may be related to cortisol levels within a certain period, reflecting the daily function of the HPA axis, while cortisol stress response 
represents the mobilization and coping ability of the HPA axis under stress (which has something in common with the CAR). This may indicate that HPA axis mobilization and abnormal coping ability may be a stable biological indicator that can indicate the disease, will not fluctuate with the severity of symptoms, and will be less affected by other factors. Therefore, it has clinical predictive significance and may even become an endophenotype.

We found that the patients with distress intolerance had more severe clinical symptoms, which also indicates that distress intolerance may represent a state that is more deviated from the healthy state, as distress intolerance in the healthy control group was very rare. However, whether distress intolerance is the result of symptoms or one of the factors that cause obvious symptoms remains to be studied.

\subsection{Negative emotions}

The difference in NA scores between patients and controls is understandable. The psychological stress challenge task is challenging, requires concentration and patience, and plays annoying stimuli in response to a mistake. This process is obviously unpleasant. The NA score of the controls increased as the task progressed, as expected. However, patients with schizophrenia were already experiencing negative emotions before starting the task. The feeling remained almost unchanged during the task, which indicated the patients with schizophrenia were less satisfied with their daily life or less satisfied with their participation in the test, relatively speaking, the content of the task was not their focus.

\subsection{Strengths and limitations}

To our knowledge, no large-sample study has previously examined the correlation between cortisol stress response and schizophrenia among the Chinese population. Possible racial differences make our research more meaningful. We collected data of more than 160 cases, providing a sample larger than those of previous studies. However, an even larger sample is needed to establish cortisol stress response as a biomarker for schizophrenia.

Cortisol levels may also be affected by other factors, such as alcohol intake or body weight. These factors were not considered in our study. Because the main focus of our study was the change of cortisol levels under stress, there was a self-control effect, and these factors would have had a limited impact on the interpretation of the results. If complete information could be obtained from the subjects, it would help improve the rigor of our conclusions. Cross-sectional studies cannot rule out differences in individual sensitivity to cortisol. Although there were self-control effects and a relatively large sample size, which can minimize the effect of sensitivity differences on the results, more information on sensitivity is needed.

Data to date have been obtained from cross-sectional studies, but disease and treatment conditions may progress or change. Therefore, long-term follow-up data are required to confirm the stability of cortisol stress response in individual treatment. 


\section{Conclusions}

Schizophrenia is a major focus of psychiatry research, and the identification of biological indicators is important for clinical diagnosis. As a potential disease diagnostic biomarker, cortisol is convenient for sampling and detection, non-invasive, and inexpensive to measure. The ability to use it alone or in combination to predict the occurrence and development of the disease is an exciting prospect.

\section{Declarations}

\section{Acknowledgements}

We are greatly indebted to all study participants, without whom this research would not have been possible.

\section{Author Contributions}

L. Elliot Hong and Yunlong Tan designed the project, provided the funding for this study and were responsible for the integrity of the data and the accuracy of the data analyses. Xiaoyu Zhu, Yu Zhu, Jinghui Tong, Junchao Huang, Yanfang Zhou, Ping Zhang and Song Chen were responsible for recruiting the patients, performing the clinical ratings, and collecting the samples. Xiaoyu Zhu wrote the paper. Xingguang Luo, Shuping Tan, Zhiren Wang, Xiaole Han, Baopeng Tian, Chiang-Shan R. Li and Li Tian were invited to form the ideas and editing the manuscript. All authors contributed to drafting the work or critically revising it for important intellectual content, and made substantial contributions to the concept and design of the study and data acquisition, analysis, and interpretation.

\section{Funding}

This work was supported by the National Natural Science Foundation of China (82171507 and 81761128021 ) and grants from the National Institutes of Health (R01MH112180 and P50MH103222).

\section{Conflict of Interest Statement}

Dr. Hong has received or is planning to receive research funding or consulting fees from Mitsubishi, Your Energy Systems LLC, Neuralstem, Taisho, Heptares, Pfizer, Sound Pharma, Luye Pharma, Takeda, and Regeneron. Other authors declare that the research was conducted without any relationship that could be interpreted as a potential conflict of interest or financial conflict.

\section{References}

1. Zubin J, Spring B. Vulnerability-a new view of schizophrenia. J Abnorm Psychol. 1977. 86(2): 103.

2. Nuechterlein KH, Dawson ME. A Heuristic Vulnerability/Stress Model of Schizophrenic Episodes. 1984 . 
3. Ciompi L. The dynamics of complex biological-psychosocial systems: Four fundamental psychobiological mediators in the long-term evolution of schizophrenia. Br J Psychiatry. 1989. 155(5): 1521.

4. Walker E, Mittal V, Tessner K. Stress and the hypothalamic pituitary adrenal axis in the developmental course of schizophrenia. Annu Rev Clin Psychol. 2008. 4(1): 189.

5. Phillips LJ, Mcgorry PD, Garner B, Thompson KN, Pantelis C, Wood SJ, Berger G. Stress, the hippocampus and the HPA axis: Implications for the development of psychotic disorders. 2006. 40(9): 725-741.

6. Smyth JM, Ockenfels MC, Gorin AA, Catley D, Porter LS, Kirschbaum C, Hellhammer DH, Stone AA. Individual differences in the diurnal cycle of cortisol. Psychoneuroendocrinology. 1997. 22(2): 89105.

7. Day FL, Valmaggia LR, Mondelli V, Papadopoulos A, Papadopoulos I, Pariante CM, Mcguire P. Blunted cortisol awakening response in people at ultra high risk of developing psychosis. Schizophr Res. 2014. 158(1-3): 25-31.

8. Fries E, Dettenborn L, Kirschbaum C. The cortisol awakening response (CAR): facts and future directions. Int J Psychophysiol. 2009. 72(1): 67-73.

9. Zorn JV, Remmelt RS, Boks MP, René S, Joëls M, Vinkers CH. Cortisol stress reactivity across psychiatric disorders: A systematic review and meta-analysis. Psychoneuroendocrinology. 2017. 77 : 25-36.

10. Girshkin L, Matheson SL, Shepherd AM, Green MJ. Morning cortisol levels in schizophrenia and bipolar disorder: a meta-analysis. Psychoneuroendocrinology. 2014. 49(1): 187-206.

11. Nugent KL, Chiappelli J, Sampath H, Rowland LM, Thangavelu K, Davis B, Du X, Muellerklein F, Daughters S, Kochunov P. Cortisol Reactivity to Stress and Its Association With White Matter Integrity in Adults With Schizophrenia. Psychosom Med. 2015. 77(7): 733-42.

12. Girshkin L, O'Reilly N, Quidé Y, Teroganova N, Rowland JE, Schofield PR, Green MJ. Diurnal cortisol variation and cortisol response to an MRI stressor in schizophrenia and bipolar disorder.

Psychoneuroendocrinology. 2016. 67: 61-69.

13. Braehler C, Holowka D, Brunet A, Beaulieu S, Baptista T, Debruille JB, Walker CD, King S. Diurnal cortisol in schizophrenia patients with childhood trauma. Schizophr Res. 2005. 79(2): 353-354.

14. Mondelli V, Dazzan P, Hepgul N, Forti MD, Aas M, D'Albenzio A, Nicola MD, Fisher H, Handley R, Marques TR. Abnormal cortisol levels during the day and cortisol awakening response in firstepisode psychosis: The role of stress and of antipsychotic treatment. Schizophr Res. 2010. 116(2): 234-242.

15. Monteleone P, Filippo CD, Fabrazzo M, Milano W, Martiadis V, Corrivetti G, Monteleone AM, Maj M. Flattened cortisol awakening response in chronic patients with schizophrenia onset after cannabis exposure. Psychiatry Res. 2014. 215(2): 263-267.

16. Bicikova M, Hampl R, Hill M, Ripova D, Mohr P, Putz Z. Neuro- and immunomodulatory steroids and other biochemical markers in drug-naive schizophrenia patients and the effect of treatment with 
atypical antipsychotics. Neuro Endocrinol Lett. 2011. 32(2): 141.

17. Mondelli V, Ciufolini S, Belvederi MM, Bonaccorso S, Di FM, Giordano A, Marques TR, Zunszain PA, Morgan C, Murray RM. Cortisol and Inflammatory Biomarkers Predict Poor Treatment Response in First Episode Psychosis. Schizophr Bull. 2015. 41(5): 1162-1170.

18. Tessner KD. Cortisol responses of healthy volunteers undergoing magnetic resonance imaging. Hum Brain Mapp. 2010. 27(11): 889-895.

19. Corcoran CM, Smith C, Mclaughlin D, Auther A, Malaspina D, Cornblatt B. HPA axis function and symptoms in adolescents at clinical high risk for schizophrenia. Schizophr Res. 2012. 135(1-3): 170174.

20. Walker EF, Trotman HD, Pearce BD, Addington J, Cadenhead KS, Cornblatt BA, Heinssen R, Mathalon DH, Perkins DO, Seidman LJ. Cortisol Levels and Risk for Psychosis: Initial Findings from the North American Prodrome Longitudinal Study. Biol Psychiatry. 2013. 74(6): 410-417.

21. Cullen AE, Zunszain PA, Dickson H, Roberts RE, Fisher HL, Pariante CM, Laurens KR. Cortisol awakening response and diurnal cortisol among children at elevated risk for schizophrenia: Relationship to psychosocial stress and cognition. Psychoneuroendocrinology. 2014. 46(100): 1-13.

22. Karanikas E, Garyfallos G. Role of cortisol in patients at risk for psychosis mental state and psychopathological correlates: A systematic review. Psychiatry \& Clinical Neurosciences. 2015. 69(5): 268-282.

23. Collip D, Habets P, Marcelis M, Gronenschild E, Lataster T, Lardinois M, Nicolson NA, Myin-Germeys I. Hippocampal volume as marker of daily life stress sensitivity in psychosis. Psychol Med. 2013. 43(7): 1377-1387.

24. Nejtek VA. High and low emotion events influence emotional stress perceptions and are associated with salivary cortisol response changes in a consecutive stress paradigm. Psychoneuroendocrinology. 2002. 27(3): 337-352.

25. Chiappelli J, Pocivavsek A, Nugent KL, Notarangelo FM, Kochunov P, Rowland LM, Schwarcz R, Hong LE. Stress-induced increase in kynurenic acid as a potential biomarker for patients with schizophrenia and distress intolerance. JAMA Psychiatry. 2014. 71(7): 761-8.

26. Watson D, Clark LA, Tellegen A. Development and validation of brief measures of positive and negative affect: the PANAS scales. J Pers Soc Psychol. 1988. 54(6): 1063-70.

27. Kay SR, Fiszbein A, Opler LA. The positive and negative syndrome scale (PANSS) for schizophrenia. Schizophr Bull. 1987. 13(2): 261-76.

28. Reynolds RM, Fischbacher C, Bhopal R, Byrne CD, White M, Unwin N, Walker BR. Differences in cortisol concentrations in South Asian and European men living in the United Kingdom. Clin Endocrinol (Oxf). 2006. 64(5): 530-4.

29. Deer LK, Shields GS, Ivory SL, Hostinar CE, Telzer EH. Racial/ethnic disparities in cortisol diurnal patterns and affect in adolescence. Dev Psychopathol. 2018. 30(5): 1977-1993.

30. Boileau K, Barbeau K, Sharma R, Bielajew C. Ethnic differences in diurnal cortisol profiles in healthy adults: A meta-analysis. Br J Health Psychol. 2019. 24(4): 806-827. 
31. Urizar GG Jr, Yim IS, Kofman YB, Tilka N, Miller K, Freche R, Johnson A. Ethnic differences in stressinduced cortisol responses:Increased risk for depression during pregnancy. Biol Psychol. 2019. 147: 107630.

32. Wang SW, Lau AS. Ethnicity moderates the benefits of perceived support and emotional expressivity on stress reactivity for Asian Americans and Euro Americans. Cultur Divers Ethnic Minor Psychol. 2018. 24(3): 363-373.

33. Bodas J, Ollendick TH. Test anxiety: a cross-cultural perspective. Clin Child Fam Psychol Rev. 2005. 8(1): 65-88.

34. Bodas J, Ollendick TH, Sovani AV. Test anxiety in Indian children: a cross-cultural perspective. Anxiety Stress Coping. 2008. 21(4): 387-404.

35. Havelka D, Prikrylova-Kucerova H, Prikryl R, Ceskova E. Cognitive impairment and cortisol levels in first-episode schizophrenia patients. Stress. 2016. 19(4): 383-9.

36. Ho RT, Fong TC, Wan AH, Au-Yeung FS, Chen EY, Spiegel D. Associations between diurnal cortisol patterns and lifestyle factors, psychotic symptoms, and neurological deficits: A longitudinal study on patients with chronic schizophrenia. J Psychiatr Res. 2016. 81: 16-22.

37. Nedic Erjavec G, Uzun S, Nikolac Perkovic M, Kozumplik O, Svob Strac D, Mimica N, Hirasawa-Fujita M, Domino EF, Pivac N. Cortisol in schizophrenia: No association with tobacco smoking, clinical symptoms or antipsychotic medication. Prog Neuropsychopharmacol Biol Psychiatry. 2017. 77: 228235.

38. Zhang XY, Zhou DF, Cao LY, Wu GY, Shen YC. Cortisol and cytokines in chronic and treatmentresistant patients with schizophrenia: association with psychopathology and response to antipsychotics. Neuropsychopharmacology. 2005. 30(8): 1532-8.

39. lancu I, Tchernihovsky E, Maayan R, Poreh A, Dannon P, Kotler M, Weizman A, Strous RD. Circulatory neurosteroid levels in smoking and non-smoking chronic schizophrenia patients. Eur Neuropsychopharmacol. 2007. 17(8): 541-5.

\section{Figures}

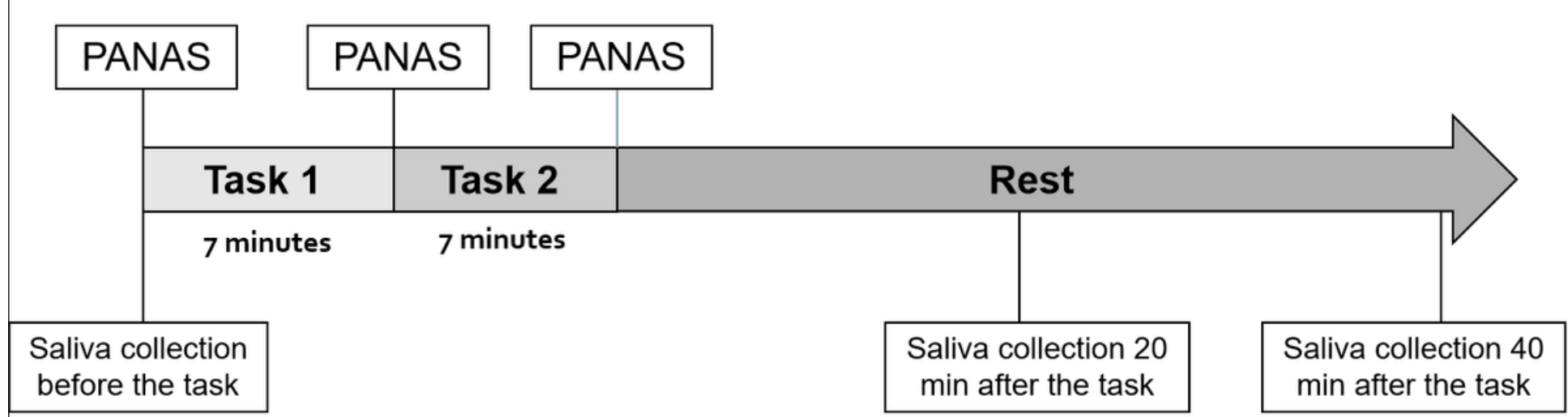


Outline of the experimental test

Figure 2

Salivary cortisol levels and NA scores of patients and controls

\section{Figure 3}

Analysis related to distress tolerance $\left({ }^{\star} \mathrm{p}<0.05\right)$ 